\title{
EFFICIENT STRATEGY FOR LOW NOX COMBUSTOR DESIGN IN THE SPATIAL DOMAIN USING MULTI-FIDELITY SOLUTIONS
}

\author{
Moresh J. Wankhede*, Neil W. Bressloff", Andy J. Keane ${ }^{\dagger}$ \\ Rolls-Royce UTC for Computational Engineering \\ University of Southampton \\ Southampton SO17 1BJ, United Kingdom
}

\begin{abstract}
Gas turbine combustor designers now routinely use highfidelity reactive computational fluid dynamics (CFD) analyses to gain valuable insight into the complex reactive flow-field and pollutant formation process. But, a large number of such computationally expensive CFD analyses are generally required to arrive at an acceptable combustor configuration. Therefore, given the practical limits on available computational resources and time, traditional combustor design methodologies using only high-fidelity CFD analyses need further improvement. To address this, a combustor design strategy using multifidelity coKriging response surface model (RSM) is developed and applied for the design of a two-dimensional test combustor problem in the spatial domain using steady-state Reynoldsaveraged Navier Stokes (RANS) formulation. The design and optimization problem is set-up for two geometric variables and a single-objective, NOx concentration, as it is of current interest to the combustor design community. The developed multifidelity strategy is also assessed for performance against highfidelity Kriging RSM strategy. This study demonstrates that the multi-fidelity design strategy can obtain good designs with up to ten times less effort than a full grid sampling search plan. However, the multi-fidelity co-Kriging strategy does not outperform the high-fidelity Kriging strategy for the given spatial domain problem.
\end{abstract}

\section{INTRODUCTION}

In 2001, the Advisory Council for Aeronautical Research in Europe (ACARE) laid down stringent fuel consumption and pollutant emissions targets for the year 2020 [1]. Year 2020 is not far off in terms of component development cycle times in the gas turbine industry and new stringent targets for 2050 are already under evaluation. Also, it is clear that these current and upcoming targets could only be realised by a major step change in gas turbine technologies and by developing rapid and efficient component design methodologies. Thus, the strategy employed during combustor design and development has a direct impact on the achievability of ACARE targets. The gas turbine combustor design process is known to be a challenging task of maintaining a crucial balance between a number of performance objectives - for e.g. low emissions, high power, high efficiency, low pulsations and a wide range of operating conditions [2]. Thus, the strategy employed during combustor design and development has a direct impact on the achievability of design targets within practical time limits. Due to the latest advances in computing power and higher accuracy CFD codes, high-fidelity combustion CFD is now becoming an important and regular part of a combustor design strategy [3]. Since a large number of high-fidelity CFD analyses are generally required during the combustor development phase, there is a need for a computationally efficient strategy where the search algorithm is not coupled directly to expensive CFD simulations.

The current state-of-the-art in the context of combustor design and optimization is the use of a Kriging response surface model (RSM) based design strategy; where pre-defined sets of highfidelity combustor CFD simulations within the target design space can be represented by an intermediate Kriging model on which a global search is performed. Previous studies [3-5] based on this methodology acknowledged that Kriging model based combustor design strategies could yield acceptable designs within a manageable timeframe by reducing the total

\footnotetext{
* At present: Combustion CFD Specialist at Dacolt International BV, The Netherlands, Email: Moresh.Wankhede@dacolt.com

\# Senior Lecturer, Email: N.W.Bressloff@soton.ac.uk

$\dagger$ Professor, Email: Andy.Keane@soton.ac.uk
} 
number of required high-fidelity CFD analyses. Yet, successful combustor design and optimization still largely depends on the total number of design variables, and objective and constraint functions involved. With the complexity of combustor designs and hence number of design variables expected to further increase, the Kriging based design strategy may not be able to perform efficiently within realistic time frames. Hence, the current best strategy for combustor design utilizing only highfidelity CFD analyses needs further improvement.

In this study, a combustor design strategy employing coKriging RSM technique is developed and applied for the design of a two-dimensional test combustor problem in the spatial domain using steady Reynolds-averaged Navier-Stokes (RANS) formulation. Since reduction of NOx emission is now a major driver of the next generation combustor design process $[6,7]$, the design and optimization problem here is set-up for two geometric variables and a single-objective, thermal NOx concentration. Initially, using multiple sampling plans a standard high-fidelity Kriging RSM strategy is used to find an optimal combustor design configuration for low NOx. Later, multi-fidelity co-Kriging strategy, consisting of two levels of solutions; a fast but approximate low-fidelity and an expensive but accurate high-fidelity combustor solution, is developed and used to perform combustor design optimization. To evaluate the effectiveness of the applied strategies, the total number of highfidelity CFD evaluations used is fixed. Then using a number of different starting sampling plans, the high and multi-fidelity design strategies are run to collect statistical data with mean convergence behavior used as a performance indicator. A confidence level assessment of both strategies is also performed.

\section{COMBUSTOR SPATIAL FLOW-FIELD}

The test combustor modeled for this study is the one used by Keller et al. [8] in an experimental study of mechanisms of instabilities in turbulent combustion and also used in [3] for developing combustor design strategies. It consists of an oblong rectangular cross-section to model the essential features of planar flow with a profiled backward-facing step designed to act as a flame holder. Note that the key focus in this section is to develop a qualitative understanding of the thermal NOx production mechanism and its spatial variation in the combustor, which is to be used as the objective function in the design studies considered later.

Figure 1 shows the computational domain used for steady RANS CFD modeling of the combustor, indicating locations of key features and inlet and outlet boundary conditions. The computational domain uses appropriate boundary conditions at the inlet and outlet of the system representing completely mixed propane and air mixture and uniform velocity at the entrance to the test section (i.e. combustion chamber). In addition to the original test setup, cooling holes are provided near the outlet, both at the upper and the lower wall, of the combustor for a realistic gas turbine combustor representation.

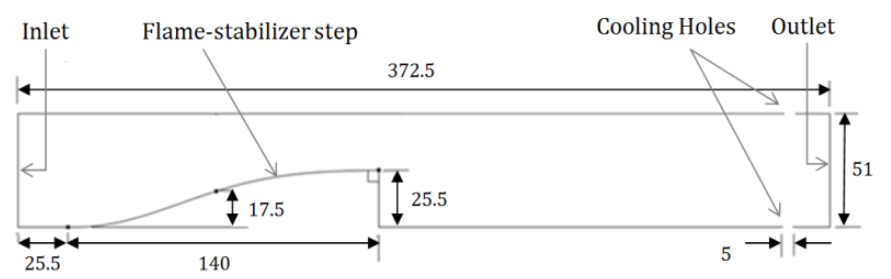

Fig. 1. 2D computational domain of the combustor with a flame-stabilizer step (All dimensions in $\mathrm{mm}$ ) $[3,8]$

Table 1. ANSYS FLUENT 12.1 CFD setup parameters

Solver: Pressure based

Space: 2D (Second-order accurate)

Energy equation: Yes

Turbulence model: $\quad k-\varepsilon$ (standard wall functions)

Transport and reaction model:

Species Partially premixed combustion

Mixture properties PDF-mixture (propane + air)

Equivalence ratio 0.86

NOx model

Formation pathways Thermal NOx only

(Zeldovich mechanism)

[O] model Equilibrium

[OH] model Equilibrium

Turbulence interaction Temperature PDF mode

Boundary conditions:

Inlet Velocity-inlet

Inlet (Momentum) $\quad \mathrm{V}_{\text {in }}=13.3 \mathrm{~m} / \mathrm{s}$

Inlet (Temperature): $300 \mathrm{~K}$

Outlet: Outflow

Cooling inlet: Velocity inlet; $\mathrm{V}_{\text {in }}=13.3 \mathrm{~m} / \mathrm{s}$

Reynolds number: $2.06 * 10^{4}$ (based on step height)

Operating pressure: $101325 \mathrm{~Pa}$

The main parameters employed in the commercial CFD package ANSYS FLUENT 12.1 are detailed in Table 1. The solver used is pressure based and employs a second-order discretization scheme for space. The standard $k-\varepsilon$ model is used for turbulence modeling, with standard wall functions. The SIMPLE pressure-correction method is used for pressurevelocity coupling. The combustion process is lean with an equivalence ratio of 0.86 . The partially premixed combustion model in ANSYS FLUENT is used as the species model which solves a transport equation for the mean reaction progress variable $\bar{C}$, (to determine the position of the flame front), as well as the mean mixture fraction $\bar{f}$, and the mixture fraction variance $\overline{f^{\prime 2}}$. For NOx, only thermal NOx formation is considered, as it is the predominant mechanism of overall NOx production in distillate-oil or gas fired turbines [9]. An 
equilibrium assumption is considered for both [O] and [OH] radicals which are used to compute the formation rate of NO. This is mainly to keep the overall computation cost lower [10] and within practical time frames. An investigation into spatial grid dependent accuracy of the CFD solution was carried out in [3] using five grid cell count refinements (mesh1: 11000, mesh2: 46000, mesh3: 190,000, mesh4: 420,000 and mesh5: $800,000)$ to evaluate a converged reactive solution. Eight processes were used in parallel on a cluster of Intel quad core processors with $2.8 \mathrm{GHz}$ clock rate. As shown in Figure 2, from an engineering design optimization perspective, mesh 3 accuracy was deemed suitable to be used for high-fidelity CFD analysis and design optimization.

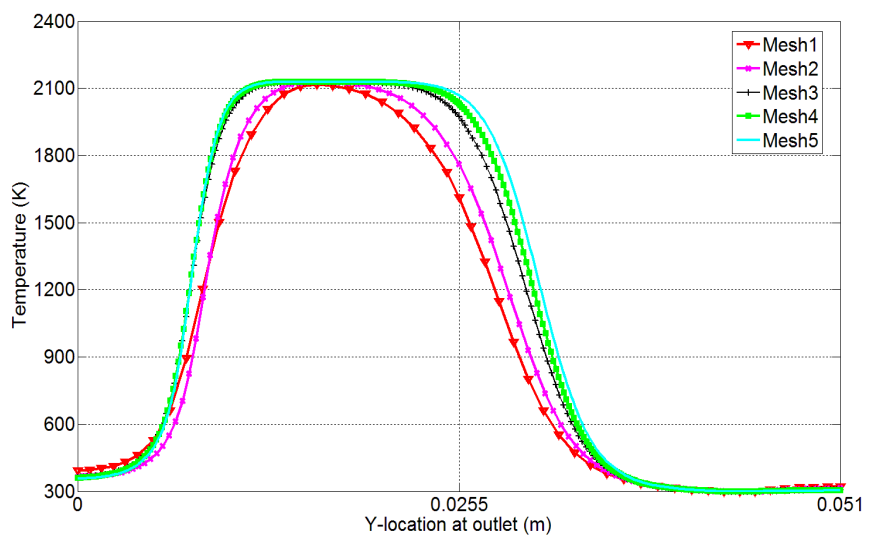

Fig. 2. Effect of spatial grid refinement on combustor outlet temperature profile as captured using steady RANS [3]

The spatial variation in the combustor flow-field captured using steady RANS is shown in Figure 3. The primary function of the flame-stabilizer step is to provide a low-velocity region for flame stabilization and combustion. The steady turbulent flames require flame stabilization mechanisms [11]. The recirculation zone behind the step provides the low-speed region necessary for flame stabilization. Figure 3(a) shows the progress variable and indicates the position of the flame surface (or flame front) inside the chamber. As the Reynolds number of the flow is in the turbulent regime, the mixture burns only in the location where the turbulent flame speed $S_{T}$ is able to sustain the mixture velocity $\tilde{u}$, i.e. the region behind the step. Therefore the chamber behind the step is separated into unburnt and burnt mixture regions by an interface, where combustion has started but not yet fully established. Above this surface $(\overline{\mathrm{C}}=$ 0 ), the fuel and oxidizer mixture is mixed but unburnt, and below this surface $(\overline{\mathrm{C}}=1)$, the mixture is completely burnt. Thus, due to high mixture velocity in the upper part of the chamber behind the step, much of the mixture escapes unburnt from the combustor. Figure 3(b) shows the temperature field inside the combustor. The temperature is maximum $(\sim 2100 \mathrm{~K})$ in the burnt mixture region, reduces in the interface region and is the lowest in the unburnt mixture region. The temperature of the mixture which escapes unburnt from the combustor remains at the inlet temperature of $300 \mathrm{~K}$. Figure 4 shows the outlet temperature profile of the combustor as captured by steady RANS. The NOx analysis in the spatial domain is performed by post-processing the previously computed reactive flow solution.

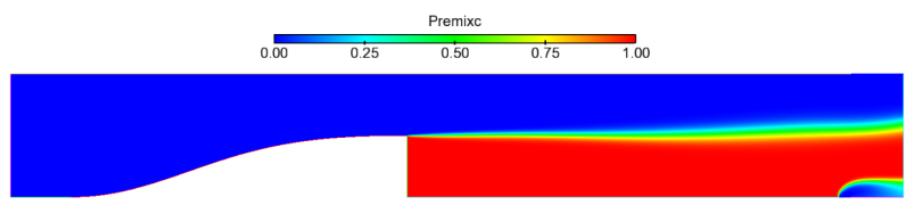

(a) Progress variable distribution

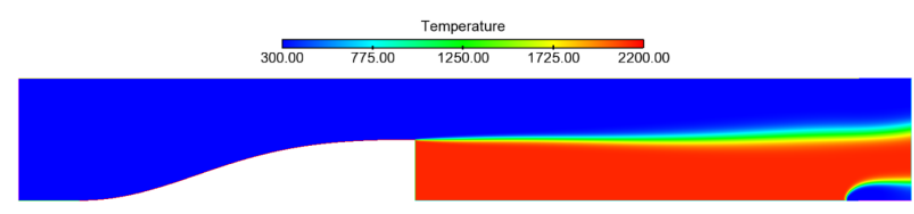

(b) Temperature distribution

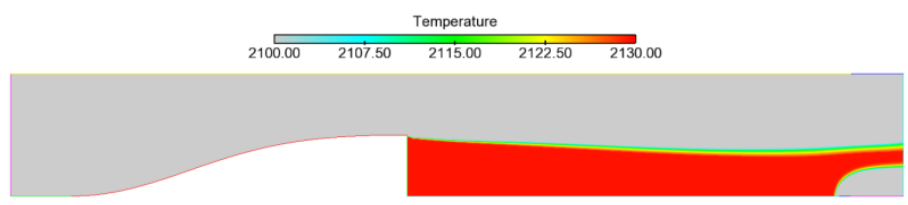

(c) Flow-field with temperature above $2100 \mathrm{~K}$

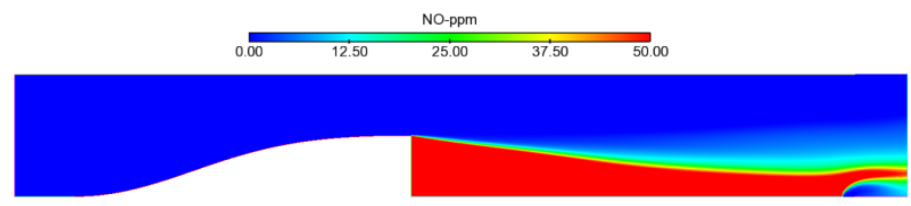

(d) Spatial distribution of NO in ppm

Fig. 3. Spatial variation in combustor flow-field captured using steady RANS analysis

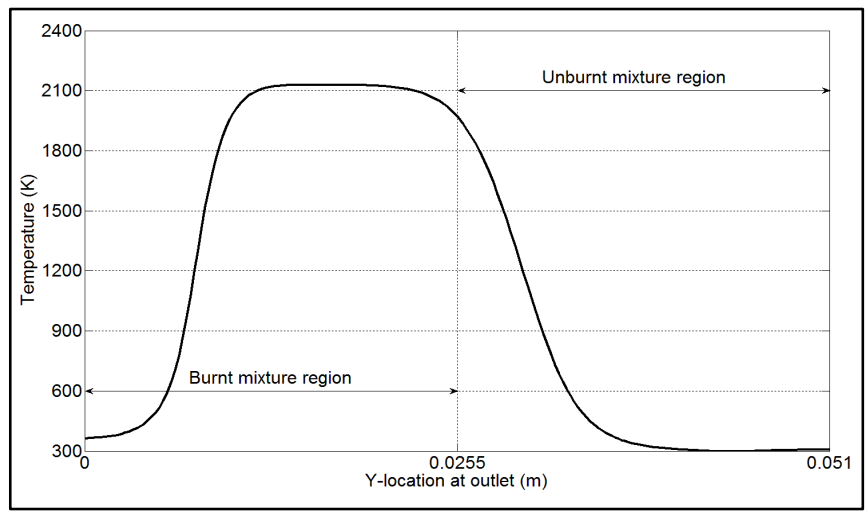

Fig. 4. Outlet temperature profile of the combustor

With combustion and fluid dynamics model turned off, only the NOx model is run until thermal NO residual convergence. Figure 3(d) shows the NO flow-field as captured by the steady RANS simulation. It shows significant thermal NO production behind the flame stabilizer step due to the occurrence of reaction processes at very high temperatures. This is in 
agreement with the Zeldovich mechanism of thermal NO formation [12, 13]. Figure 3(c) shows the part of the reactive flow-field where the temperature is the highest $(>2100 \mathrm{~K})$ which correlates to the high thermal NO concentration zone in Figure 3(d). Figure 5 shows the outlet thermal NO ( $\mathrm{ppm})$ profile of the combustor captured by steady RANS.

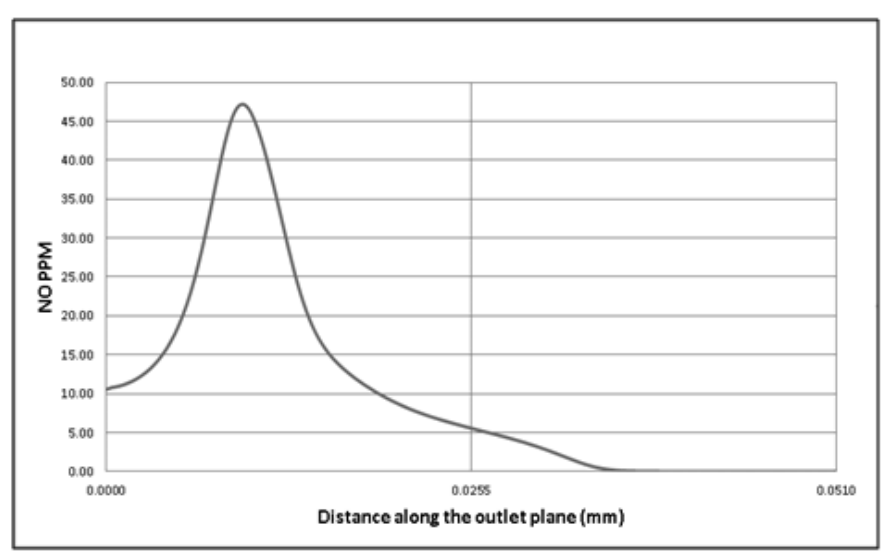

Fig. 5. Outlet thermal NO profile of the combustor

\section{SPATIAL DOMAIN DESIGN PROBLEM}

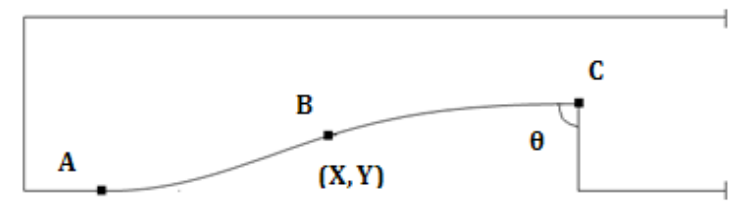

Fig. 6. Flame-stabilizer step design parameterization using spline control points

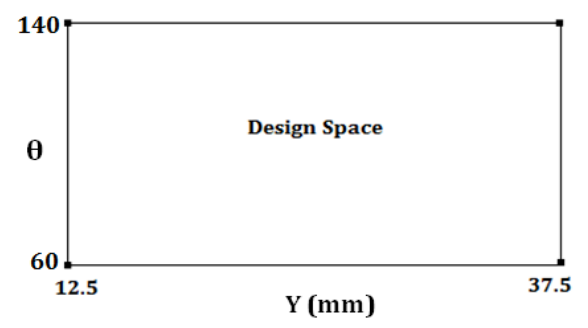

Fig. 7. Design space with lower and upper bounds

The construction of the 2D profiled backward-facing step combustor in Figure 1 is carried out using a cubic spline within the CAD package CATIA (Computer Aided Three Dimensional Interactive Application) version V5R18. CATIA is a multiplatform CAD/CAM/CAE commercial software suite developed by the French company Dassault Systemes [14]. A cubic spline is a spline constructed of piecewise thirdorder polynomials which passes through a set of control points. Consider a 1-dimensional spline for a set of $n+1$ points $\left(\mathrm{y}_{0}, \mathrm{y}_{1} \ldots \mathrm{y}_{\mathrm{n}}\right)$ with $\mathrm{n}$ intervals between them. There is a separate cubic polynomial for each interval, each with its own coefficients

$$
Y_{i}(x)=a_{i}+b_{i} x+c_{i} x^{2}+d_{i} x^{3}
$$

Together, these polynomial segments are denoted as $\mathrm{Y}(\mathrm{x})$, the spline, as shown in Equation 1, where, $\mathrm{x}$ is a parameter $\mathrm{x} C\left[\mathrm{x}_{\mathrm{i}}\right.$, $\left.\mathrm{x}_{\mathrm{i}+1}\right]$ and $\mathrm{i}=0, \ldots \mathrm{n} . \mathrm{a}_{\mathrm{i}}, \mathrm{b}_{\mathrm{i}}, \mathrm{c}_{\mathrm{i}}$ and $\mathrm{d}_{\mathrm{i}}$ are the constraints.

Figure 6 shows a closer view of the flame stabilizer step (A-BC) baseline geometry. Points A, B and C are connected by a spline curve of which control point $\mathrm{A}$ and $\mathrm{C}$ are fixed. However, the angle $\theta$ at point $\mathrm{C}$ is free. At control point $\mathrm{B}$, the $\mathrm{X}$-coordinate is fixed at a distance of $95.5 \mathrm{~mm}$ from the inlet and the $y$-coordinate is variable. Thus, two variables [Y and $\theta$ ] are used to change the shape of the flame-stabilizer step and thus influence flame/vortex interaction processes downstream. A baseline spline is defined by $\mathrm{Y}=17.5 \mathrm{~mm}$ and $\theta=90$ degrees. Figure 7 shows the two-dimensional design space that is considered for the design study indicating the upper and lower limits of the variable values.

With the optimisation parameters and design space defined, a reasonably accurate representation of the design space is evaluated on a 10x10 regular grid of design points for outlet thermal NO objective function in spatial domain. The spatial domain objective function for combustor outlet $\mathrm{NOx}$ is concerned with thermal NO concentration in parts per million (ppm) at the outlet plane. For this prediction a custom field function in ANSYS FLUENT 12.1 is setup, which computes NO ppm from the following equation [10]:

$$
\text { NO ppm }=\frac{\text { NO mole fraction } \times 10^{6}}{1-\mathrm{H}_{2} \mathrm{O} \text { mole fraction }}
$$

An area-weighted average of the thermal NO in ppm at the outlet plane is considered as the objective function to be minimized in the optimisation process.

Figure 8 provides a relatively accurate map of the objective function landscape, constructed using a 10x10 regular grid of CFD evaluations data of steady outlet thermal NO and Kriging RSM. A valley of lower objective function values is observed at higher values of $Y$ and intermediate values of $\theta$, indicating a region of good designs. This valley becomes the area of attention when applying Kriging optimization strategy for steady outlet NO. Figure 9 shows the best and worst designs for outlet thermal NO objective function when using a 10x10 regular grid of CFD evaluations. The best design [c.f. Figure 9(a)] is obtained at high values of $\mathrm{Y}$ and intermediate values of $\theta$. The amount of thermal NO produced is low mainly due to the small recirculation zone behind the step [c.f. Figure 10(a)] and is entrapped. Hence, a low amount of thermal NO is transported towards the outlet. However, in the case of worst design [c.f. Figure 9(b)], the thermal NO production is larger due to bigger recirculation vortex behind the step [c.f. Figure $10(b)]$. Therefore, a smaller recirculation vortex behind the step favors good designs with lower thermal NO. 


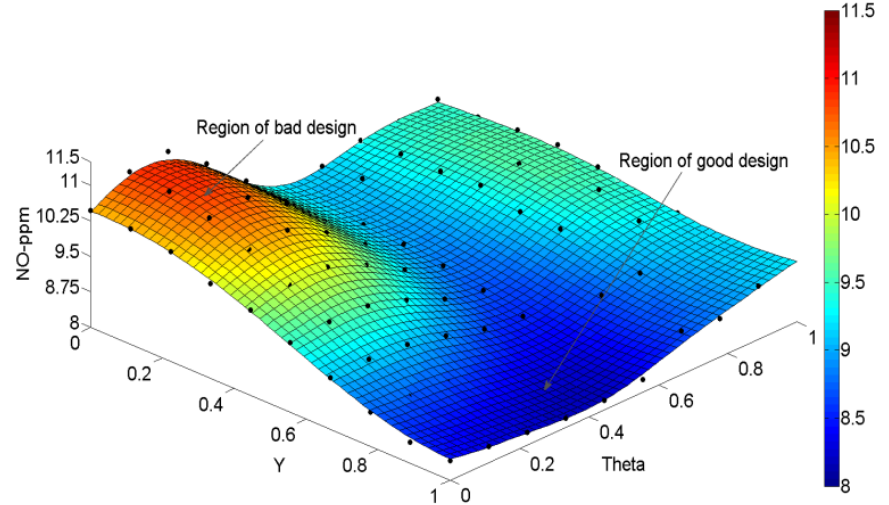

Fig. 8. Kriging response surface for steady outlet thermal NO generated using $10 \times 10$ regular grid CFD data

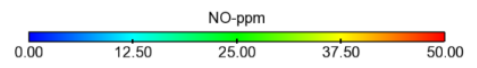

(a) Best design $[\mathrm{Y}=0.89, \theta=0.44, \mathrm{NO}=8.170 \mathrm{ppm}]$

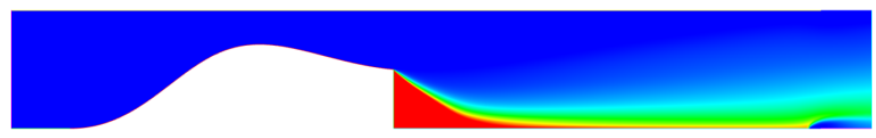

(b) Worst design $[\mathrm{Y}=0, \theta=0.22, \mathrm{NO}=10.980 \mathrm{ppm}]$

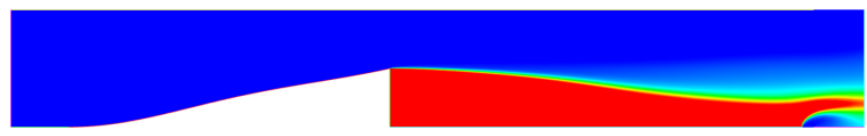

Fig. 9. Best and worst designs for outlet thermal NO obtained using 10x10 regular grid CFD evaluations

(a) Best design

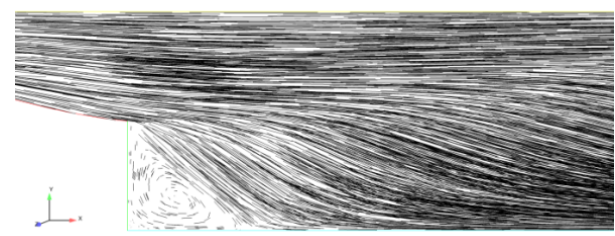

(b) Worst design

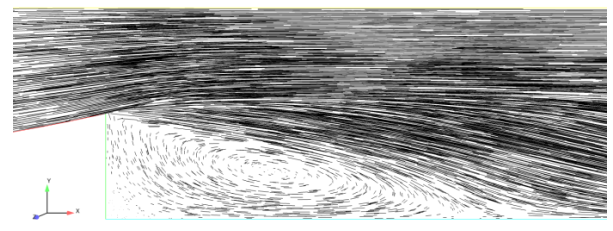

Fig. 10. Vortex behind the best and worst step designs for outlet thermal NO

\section{KRIGING RSM BASED DESIGN STRATEGY}

The existing strategies that use response surfaces for design and optimization can be classified on the basis of the type of response surface and the method that is used to search for the update points. Jones [15] provides an excellent description of various types of response surface methods that are currently used. The Kriging interpolation method was first developed by Daniel Krige [16] as a geostatistical technique to estimate unknown values from data observed at known locations. More information and detailed derivation of the Kriging prediction methodology is given in Sacks et al. [17].

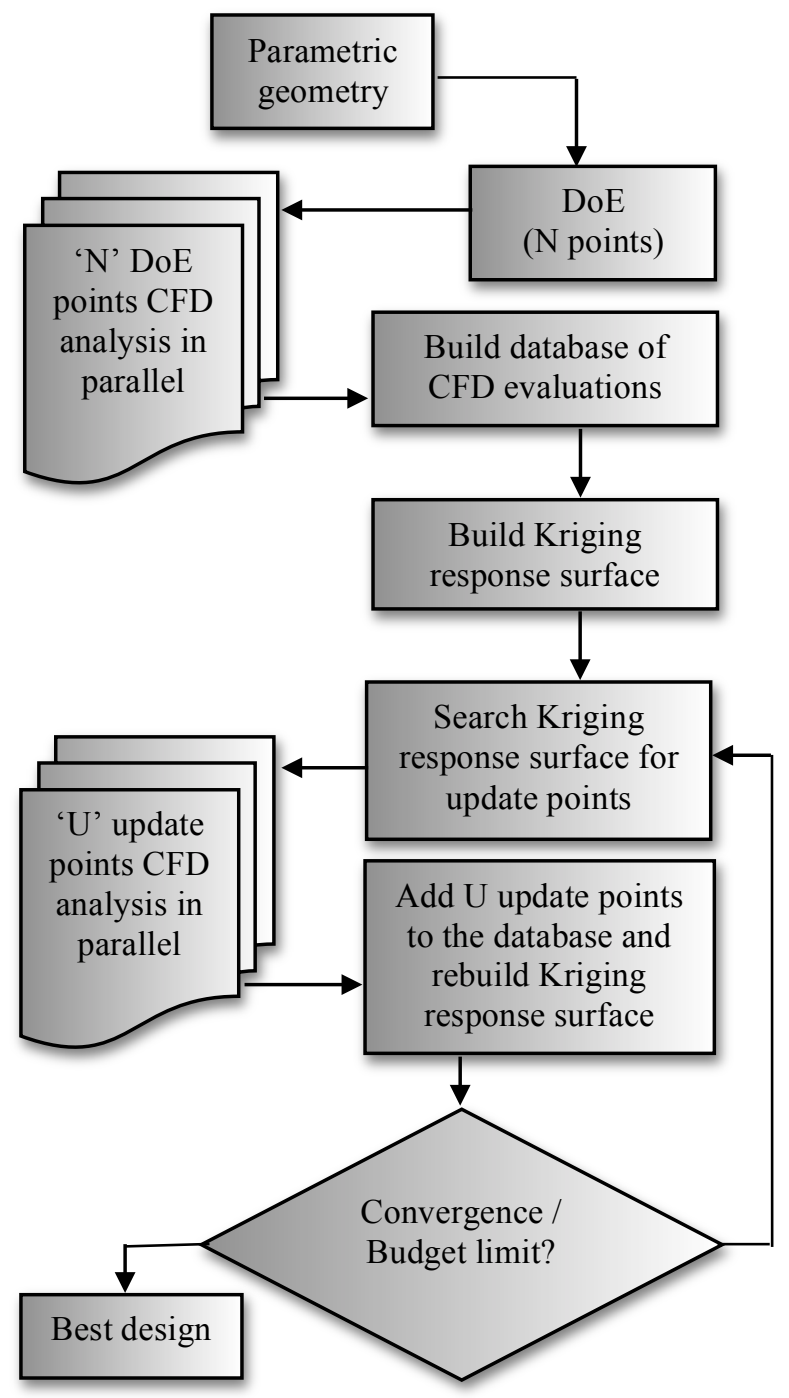

Fig. 11. Kriging response surface model based high-fidelity combustor design strategy

Figure 11 shows the Kriging RSM based high-fidelity combustor design strategy. The three key stages of the strategy are: (1) Initial sampling using DOE (2) Constructing Kriging response surface and (3) Update points search strategies for increasing Kriging prediction accuracy. Identifying the effects of variables or parameters within a design space, a space filling design of experiments (DOE) method is used to generate an initial sample of evaluation points. These DOE points are evaluated in parallel using CFD analysis. A database of objective function values at sample points obtained using CFD evaluations is built. 


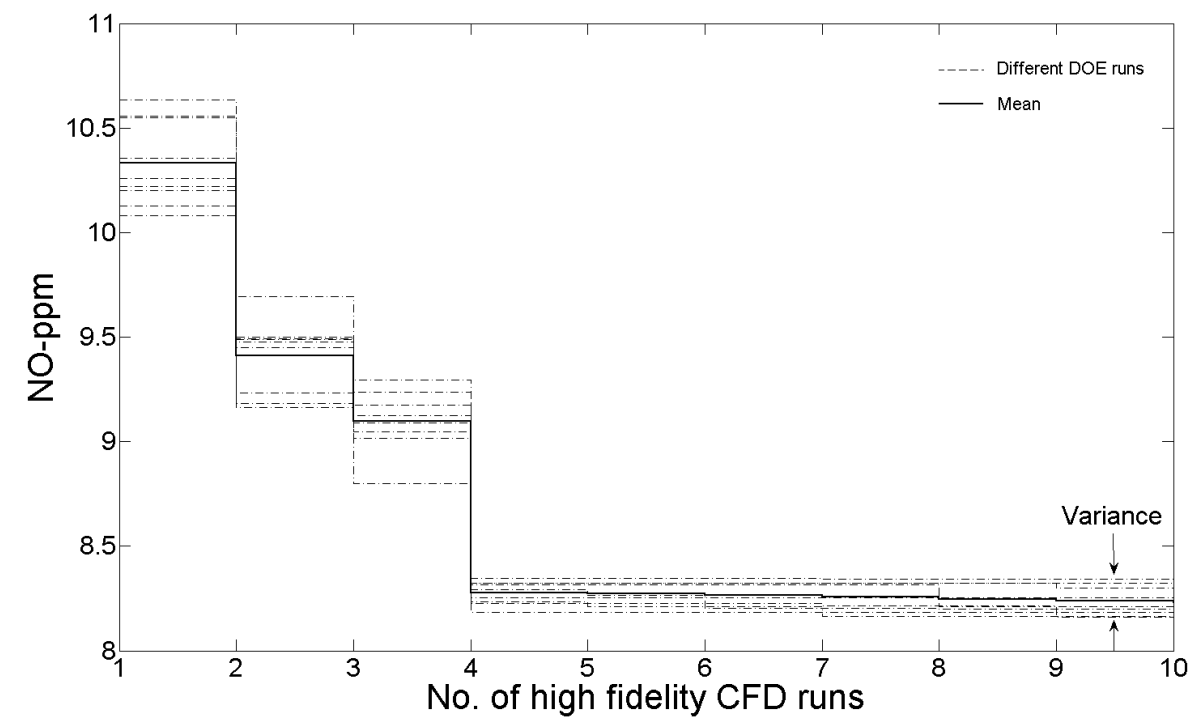

Fig. 12. Steady outlet thermal NO optimization search histories over a fixed computational budget of 10 high-fidelity CFD runs (4 in initial sample +6 updates)

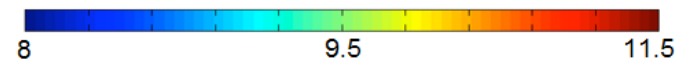

Kriging RSM consisting of best overall design amongst different search histories

(a) After DOE

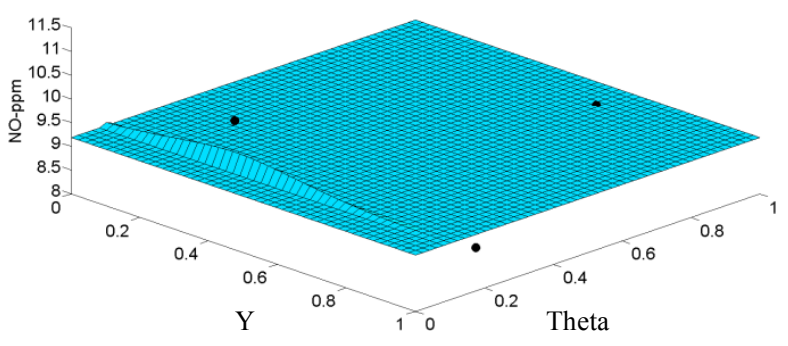

Kriging RSM consisting of worst design amongst different search histories

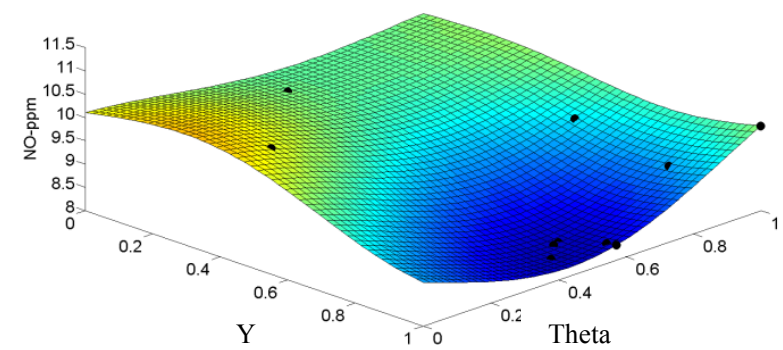

(c) After DOE

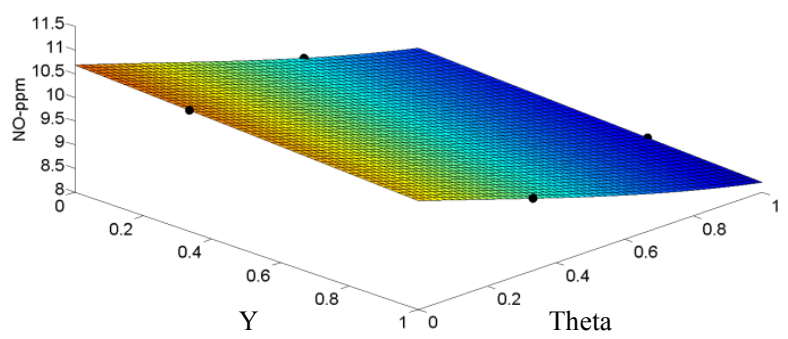

(d) After DOE + Updates

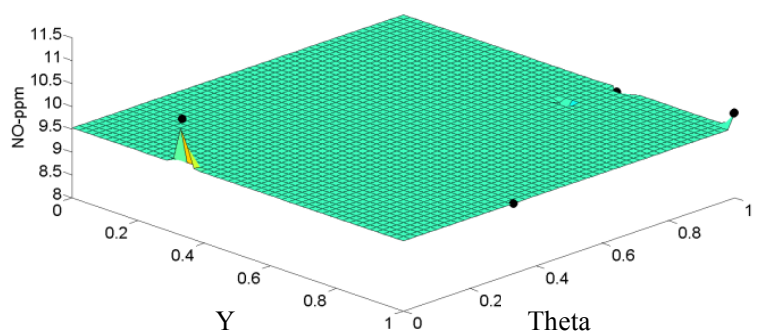

Fig. 13. Kriging response surfaces consisting of best and worst designs for steady outlet thermal NO (ppm) 
Then, a Kriging response surface model is constructed based on the observations at sample points within the design space. This surface provides an initial prediction of the variation of objective function values in the design space. As the response surface model accuracy is limited due to a relatively small initial sample, the accuracy of the model is increased by adding further update points. For the design optimization strategy to be efficient, both exploration and exploitation of the design space is necessary in order to search interesting design configurations and improve the quality of the RSM. Simultaneously, the available information must be used to rapidly converge to a global optimum. Here, the update points are found using a genetic algorithm followed by dynamic hill climbing algorithm, which provides a combination of global and local search strategy to find the exact location of the predicted optimum configuration. The resulting update points are again evaluated in parallel using CFD analysis and added to the database to update the Kriging model. This process is continued until the RSM is converged or the given computational budget gets exhausted.

Since using a 10x10 grid of points to sample the design space is very expensive, attention here is focused on using a small initial sample plan followed by an update strategy to locate the best design. The total computational budget for this Kriging based design strategy in the spatial domain is fixed here at 10 high-fidelity CFD runs (c.f. Table 2). For initializing the design study, 4 space-filling sample points are generated using the optimal Latin-Hypercube DOE method. After constructing the Kriging surface, based on the observations from the DOE points, two update points are generated per update cycle (c.f. Table 3). One is obtained using the best Kriging prediction criterion in the response surface and the other is obtained using the expected improvement criterion [18]. Thus a balanced exploration and exploitation approach is used to update the Kriging response surface model [18]. Further, the Kriging based design strategy (c.f. Figure 11) is applied on 9 different optimal Latin-Hypercube DOE samples to investigate their effect on the strategy's ability to find optimum design configurations within the given computational budget of 10 high-fidelity runs.

Figure 12 shows the optimization search histories for the Kriging based design strategy using 9 different DOE samples over a fixed computational budget of 10 high-fidelity CFD runs for outlet thermal NO. It also shows the mean performance of all the 9 search histories. As observed in Figure 12, the different initial samples clearly have an effect on the way the optimization process progresses. This is because different initial samples lead to different information being available at the DOE stage with altered Kriging model convergence behaviour. Hence, each optimization cycle leads to a different optimal design in Figure 12. A spread, or variation, in the search histories convergence is also shown in Figure 12. Figure 13 shows the Kriging response surfaces consisting of best and worst designs for steady outlet thermal
NO (ppm) after the DOE stage and end of the optimisation cycle stage. In comparison to the shape of the response captured using the 10x10 grid CFD evaluations (c.f. Figure 8), the response surface after DOE + Updates in Figure 13(b) captures the good and bad design regions within the given fixed computational budget. But, the response surface in Figure 13(d) fails to capture the shape of the response within the given budget. This difference is due to the quality of the information available at the respective DOE stages, which subsequently affects convergence and finding the optimal design with a limited budget. This observation is consistent with one of the possible pitfalls associated with Kriging mentioned by Jones [15].

\section{CO-KRIGING RSM BASED DESIGN STRATEGY}

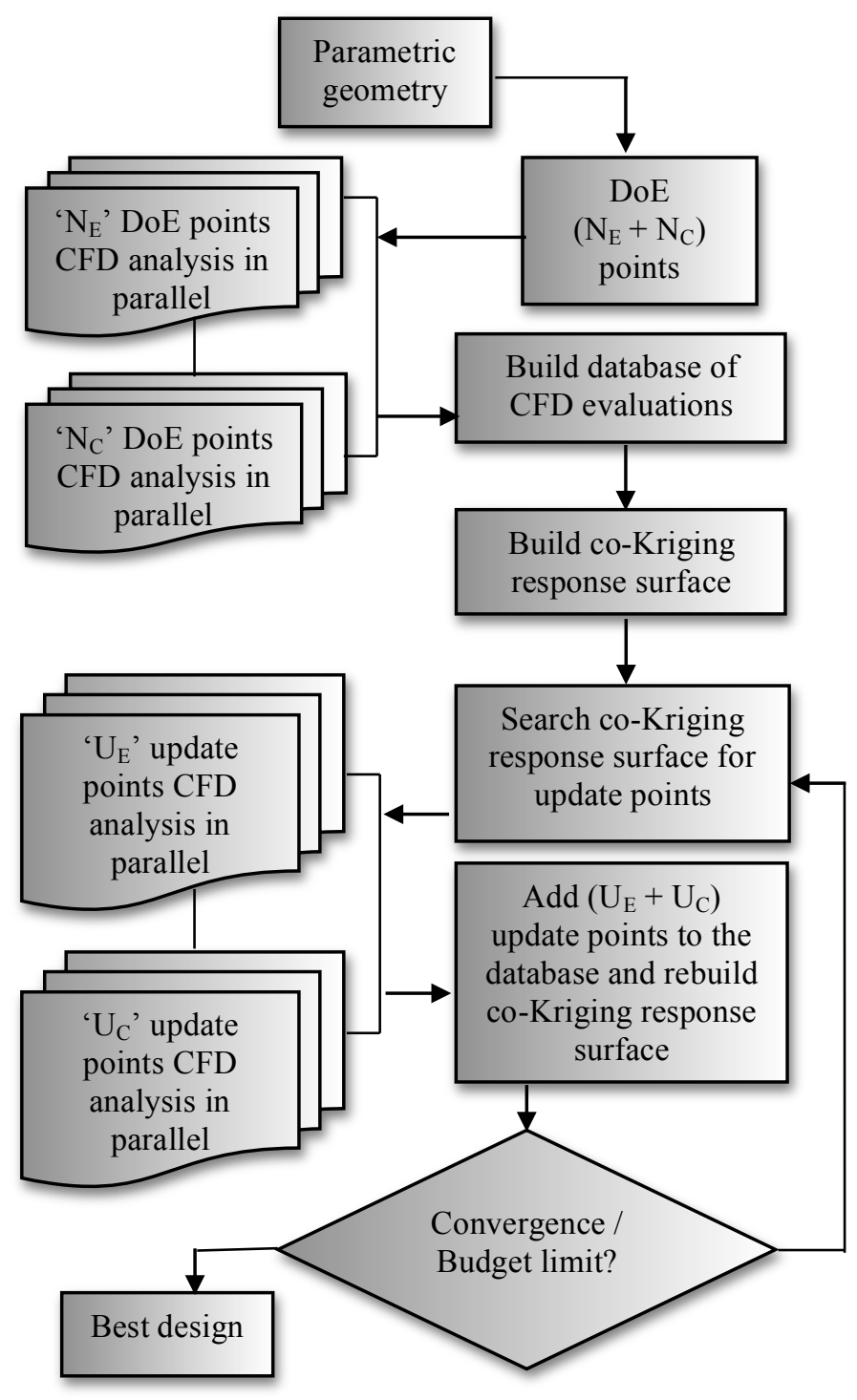

Fig. 14. Co-Kriging response surface model based multifidelity combustor design strategy 
(a)

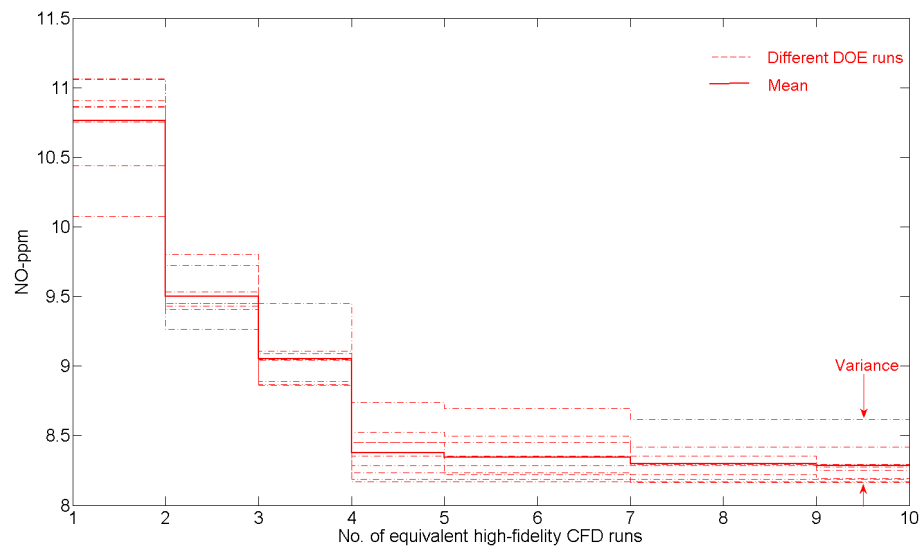

(b)

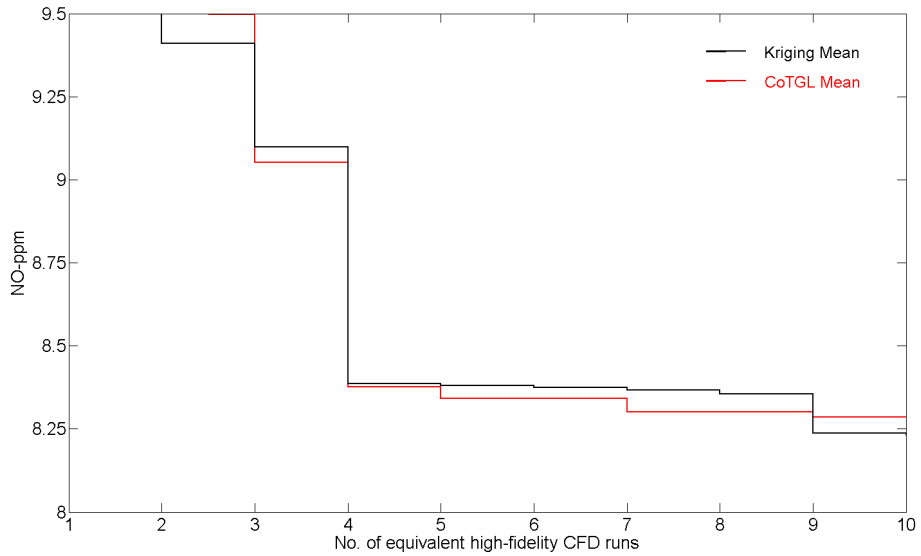

Fig. 15. (a) Optimization search history of CoTGL strategy over a fixed computational budget of $4 \mathrm{DoE}+3$ update cycle (6 update points) runs (b) Comparison between Kriging and CoTGL strategies mean performances

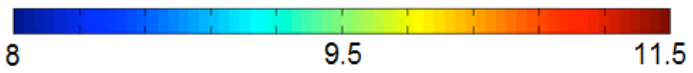

(a) After DOE

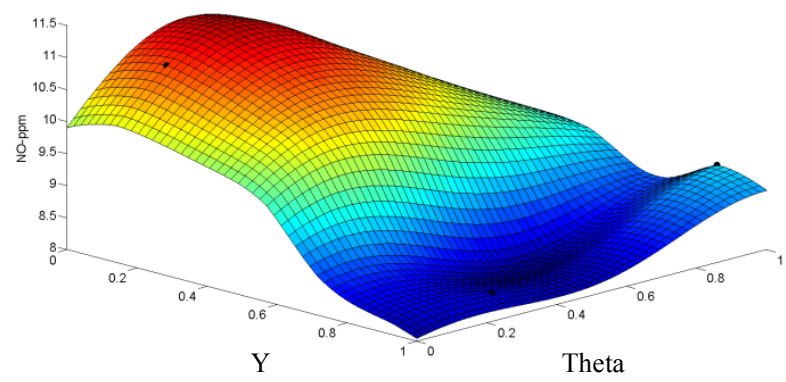

(b) After DOE + Updates

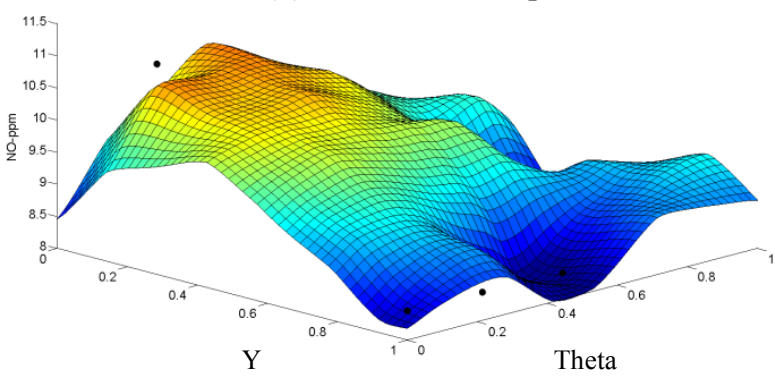

Fig. 16. CoTGL response surface consisting of best overall design for steady outlet thermal NO (ppm)

(a) CoTGL model correlation

$\left(r^{2}=0.2789\right)$

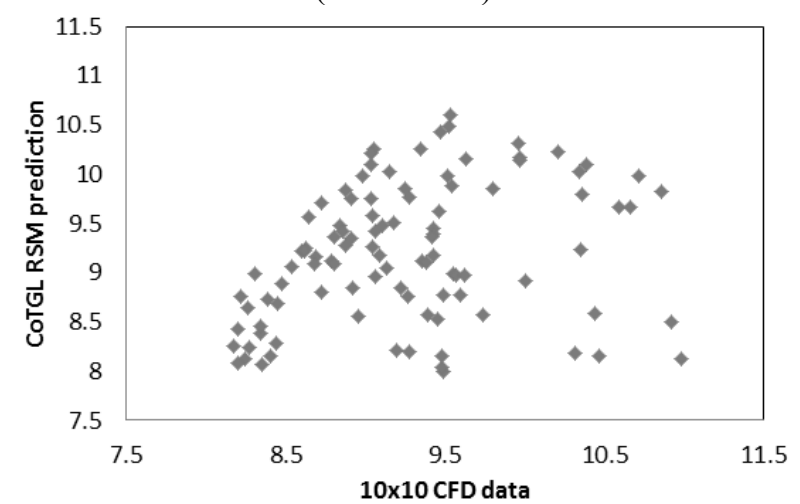

(b) Kriging model correlation $\left(r^{2}=0.8785\right)$

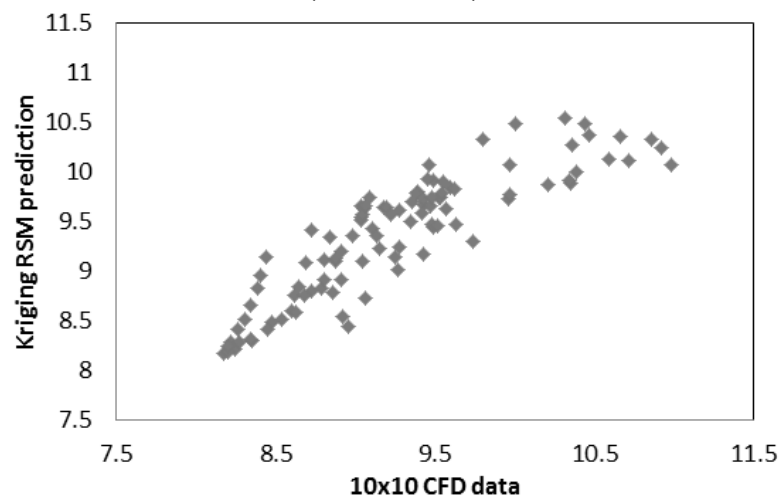

Fig. 17. Comparison between correlations of CoTGL and Kriging RSM (after DOE + Updates) predictions with 10x10 CFD data for steady outlet thermal NO (ppm) 
Table 2 Relative budgets of Kriging and co-Kriging design strategies for spatial domain outlet thermal NO optimization

\begin{tabular}{|ccccc|}
\hline Strategy & $\begin{array}{c}\text { Given budget for high- } \\
\text { fidelity CFD runs }\end{array}$ & $\begin{array}{c}\text { Total no. of high-fidelity } \\
\text { CFD runs performed }\end{array}$ & Cost ratio & $\begin{array}{c}\text { Total no. of low-fidelity CFD } \\
\text { runs performed }\end{array}$ \\
Kriging & 10 & 10 & - & - \\
CoTGL & 10 & 7 & E $\approx 10 \mathrm{C}$ & 30 \\
\hline
\end{tabular}

Table 3 High and low fidelity CFD runs budget distribution for Kriging and Co-Kriging design strategies over DOE and update cycle stage [Note: $N_{E}$ and $U_{E}$ in bold, $N_{C}$ and $U_{C}$ in round brackets, EI: expected improvement update, BP: best predicted update, ER: maximum error update]

\begin{tabular}{|ccccc|}
\hline Strategy & DOE & Update cycle 1 & Update cycle 2 & Update cycle 3 \\
Kriging & $\mathbf{4}$ & $\mathbf{2}[1 \mathrm{EI}, 1 \mathrm{BP}]$ & $\mathbf{2}[1 \mathrm{EI}, 1 \mathrm{BP}]$ & $\mathbf{2}[1 \mathrm{EI}, 1 \mathrm{BP}]$ \\
CoTGL & $\mathbf{4}(15)$ & $\mathbf{1}(5)[1 \mathrm{EI}, 2 \mathrm{BP}, 2 \mathrm{ER}]$ & $\mathbf{1}(5)[1 \mathrm{EI}, 2 \mathrm{BP}, 2 \mathrm{ER}]$ & $\mathbf{1}(5)[1 \mathrm{EI}, 2 \mathrm{BP}, 2 \mathrm{ER}]$ \\
\hline
\end{tabular}

Co-Kriging is in effect an extension of the Kriging methodology [19], which consists of correlating multiple levels of data. CFD simulations can be run at different levels of complexity, e.g. using two different levels of mesh resolution, such that there is a relatively accurate but slow analysis along with a fast but less accurate analysis. However, in the context of design optimization, these fast approximations, though somewhat inaccurate, may well include important flow-field features and can be used for design search investigation. To improve the efficiency of only high-fidelity surrogate model based design optimization systems (c.f. Figure 11), a greater quantity of fast (or cheap) analyses can be used in combination with a smaller number of expensive accurate analyses, in a multi-fidelity co-Kriging methodology, to enhance the accuracy of the high-fidelity function surrogate model at a lower computational cost [20]. More information and detailed derivation of the co-Kriging method is given in Forrester et al. $[19,20]$. Figure 14 shows a co-Kriging response surface model based design optimization strategy with $\mathbf{N}_{\mathbf{C}}$ (cheap) and $\mathbf{N}_{\mathbf{E}}$ (expensive) DOE points and $\mathbf{U}_{\mathbf{C}}$ (cheap) and $\mathbf{U}_{\mathbf{E}}$ (expensive) update points per update cycle, where $\mathbf{N}_{\mathbf{C}}>\mathbf{N}_{\mathbf{E}}$ and $\mathbf{U}_{\mathbf{C}}>\mathbf{U}_{\mathbf{E}}$. $\mathbf{N}_{\mathbf{E}}$ DOE points and $\mathbf{U}_{\mathbf{E}}$ updates are subsets of $\mathbf{N}_{\mathbf{C}}$ and $\mathbf{U}_{\mathbf{C}}$ respectively. Starting with an initial set of $\mathbf{N}_{\mathbf{C}}$ and $\mathbf{N}_{\mathbf{E}}$ DOE points, a combined database of objective function values is constructed. Based on these observations, a co-Kriging response surface model is built. Further, to increase the accuracy of the co-Kriging response surface model, update points are selected at either or all locations of the co-Kriging (a) best prediction, (b) maximum prediction error and (c) maximum expected improvement. Also, the update points $\mathbf{U}_{\mathbf{C}}$ and $\mathbf{U}_{\mathbf{E}}$ are evaluated in parallel and the co-Kriging RSM is rebuilt and searched for optimal designs. This process is iterated until response surface model convergence or the end of a given computational budget.

For co-Kriging in the spatial domain, two different levels of grid (or spatial) resolutions are used. The fine grid resolution (mesh3) is used as the expensive high-fidelity model and the coarse grid resolution (mesh1) is used as the cheap low-fidelity model. In order to apply the co-Kriging based design optimization strategy for two different grid level (CoTGL) solutions, the computational cost ratio between the cheap lowfidelity (C) and expensive high-fidelity (E) CFD solutions is used as the basis for determining the total number of CFD evaluations. Table 2 shows the details of the CoTGL strategy budget relative to the standard Kriging strategy for design optimization in the spatial domain. The distribution of the total number of high and low fidelity CFD runs over four stages (DOE and three update cycles) of the CoTGL strategy relative to the Kriging strategy is shown in Table 3 . The total number of high-fidelity CFD runs over CoTGL design optimization cycle is limited to seven. The remaining three high-fidelity runs are replaced by an equivalent number of low-fidelity runs, as determined by the cost ratio between the low and high fidelity model for CoTGL.

Figure 15(a) shows the steady outlet thermal NO optimization search histories for the CoTGL design strategy, using nine different DOE samples, over a fixed computational budget and the mean performance of all the search histories. The DOE evaluation consists of four high-fidelity and fifteen low-fidelity CFD runs. Further, the three-update cycles consist of three high-fidelity and fifteen low-fidelity CFD runs in total (c.f. Table 3). Similar to the Kriging strategy optimization histories (c.f. Figure 12), the different initial samples cause variations in CoTGL model convergence due to different information being available at the DOE stage. Hence, different optimal designs are obtained at the end of each optimization cycle. Figure 15(a) also shows the variation in the convergence across the different CoTGL experiments at the end of the budget. Figure 15(b) shows the comparison between the means of the optimization search histories for the Kriging and CoTGL strategies for steady outlet thermal NO. The CoTGL strategy does not perform better than the Kriging strategy in terms of mean convergence at the end of the fixed computational budget. However, the CoTGL mean for outlet thermal NO is below the 
Kriging mean after the DOE stage, thus indicating the CoTGL strategy's ability to find a good design earlier in the design process.

Figure 16 shows the response surfaces of the CoTGL strategy containing best optimal designs for steady outlet thermal NO. In comparison to the shape of the response surface captured using 10x10 grid CFD evaluations (c.f. Figure 8), the shape of the CoTGL response surfaces after DOE [Figure 16(a)] and updates [Figure 16(b)] appear more globally accurate, compared to the Kriging response surfaces [Figure 13(a) and 13(b)] due to the availability of a greater quantity of information from the low-fidelity model. However, the CoTGL response surface at the end of the optimization cycle [Figure 16(b)] also appears to be non-smooth due to presence of noise from the low-fidelity model. This noise is regressed in the coKriging prediction. More information on regression methodology used is provided in [19]. Figure 17 shows the comparison between the correlation of CoTGL and Kriging RSM predictions (at the end of the computational budget) with 10x10 CFD data. As per the scatter plots of Figure 17, the relationship between CoTGL RSM prediction and 10x10 CFD data for outlet thermal NO is more non-linear as compared to the relation between Kriging RSM prediction and 10x10 CFD data. The CoTGL model has $\mathrm{r}^{2}=0.278$ which is much lower than the Kriging model $\left(r^{2}=0.878\right)$. Even though visually, the shape of the CoTGL RSM appears more accurate, the presence of noise in the prediction again lowers the correlation. Table 4 shows the comparison between the best designs obtained by different spatial domain strategies for outlet thermal NO within fixed computational budget. The overall best design configuration with lowest thermal NO value is found by Kriging strategy.

\begin{tabular}{|c|c|c|c|}
\hline \multicolumn{4}{|c|}{$\begin{array}{l}\text { Table } 4 \text { Comparison between the best overall designs } \\
\text { found by different spatial domain strategies and } 10 \times 10 \\
\text { CFD data }\end{array}$} \\
\hline Strategy & $\mathrm{Y}$ & Theta & NO (ppm) \\
\hline $10 \times 10$ data & 0.890 & 0.440 & 8.170 \\
\hline Kriging & 0.933 & 0.446 & 8.160 \\
\hline CoTGL & 0.913 & 0.529 & 8.170 \\
\hline
\end{tabular}

In this study, the DOE sample size $(\mathrm{N})$ is 9 , which may or may not be large enough. However, adding more samples is expensive as the computation time of the entire design cycle is high. Hence, to reduce the uncertainty associated with the accuracy of the estimated mean without adding more samples, the confidence level is assessed here using bootstrap methodology [21] in MATLAB version R2010a. Table 5 shows the upper and lower limit values of Kriging and CoTGL strategies $95 \%$ confidence intervals for steady outlet thermal NO. Figure 18 shows the comparison between the 95\% confidence intervals on the Kriging and CoTGL means for steady outlet thermal NO. If the confidence intervals on the two sample estimates do not overlap, one can be confident that the true value (or population) of the estimate differs significantly, statistically [22]. For outlet thermal NO the confidence intervals on Kriging and CoTGL mean do overlap but not considerably. Also the lower and upper bounds of the confidence interval for the Kriging mean are narrower. Hence, a statistically significant difference would be observed on the population mean obtained by using Kriging and CoTGL strategies for outlet thermal NO.

\begin{tabular}{|c|c|c|}
\hline \multicolumn{3}{|c|}{$\begin{array}{c}\text { Table } 5 \text { Bootstrapped confidence intervals }(\mathrm{CI}) \text { for } \\
\text { Kriging and CoTGL strategies }\end{array}$} \\
\hline Strategy & Lower limit (ppm) & Upper limit (ppm) \\
\hline Kriging & 8.194 & 8.281 \\
\hline CoTGL & 8.218 & 8.431 \\
\hline
\end{tabular}

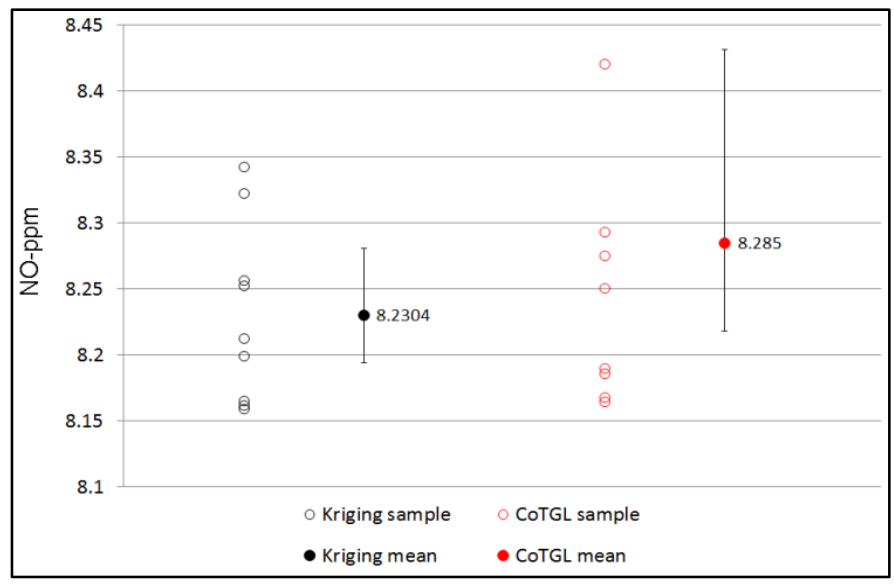

Fig. 18. Estimated means with $95 \% \mathrm{CI}$ and sample data of Kriging and CoTGL strategies for steady outlet thermal NO (ppm)

\section{CONCLUSION}

Spatial domain combustion and thermal NOx formation mechanism in a two-dimensional combustor has been qualitatively modeled using steady RANS formulation. A stable flame-front was captured behind the flame-stabilizer step in the region with low mixture velocities. In the parts of the reactive flow-field with highest temperatures $(>2100 \mathrm{~K})$, high thermal NO concentration is observed which is entrained by the vortex stabilized behind the step. Based on this analysis, outlet thermal NO was used as the objective function for developing and comparing combustor design strategies in spatial domain within a fixed computational budget. A Kriging RSM based highfidelity design strategy and co-Kriging RSM based multifidelity design strategy using two-grid level solutions- CoTGL were used to optimize the shape of a flame-stabilizer step. Both design optimization strategies were repeated on nine different initial samples, which revealed how the convergence search history varied, leading to different optimal designs. The statistics of interest i.e. mean performance over all the DOE samples showed that strategy CoTGL found a good design earlier in the design process compared to the standard Kriging 
strategy. However, using 95\% confidence level assessment, the Kriging strategy CI was much narrower and below the CoTGL strategy CI.

Hence, principally, it could be concluded that multi-fidelity CoTGL strategy does not outperform high-fidelity Kriging strategy for combustor design in the spatial domain. Nevertheless, evidence exists of finding a good design earlier in the process using CoTGL strategy compared to the Kriging strategy and full grid sampling search plan. This study forms the basis of investigation of multi-fidelity solutions based strategies for combustor design in the temporal domain.

\section{NOMENCLATURE}

$\begin{array}{ll}\overline{\mathrm{C}} & \text { : Mean reaction progress variable } \\ \bar{f} & : \text { Mean mixture fraction } \\ \overline{f^{\prime 2}} & : \text { Mean mixture fraction variance } \\ \Phi & : \text { Equivalence ratio } \\ \text { ACARE } & : \text { Advisory Council for Aeronautical Research in } \\ & \text { Europe } \\ \text { CFD } & : \text { Computation Fluid Dynamics } \\ \text { CI } & \text { : Confidence Interval } \\ \text { CoTGL } & : \text { Co-Kriging using Two Grid Levels } \\ \text { DOE } & \text { : Design of Experiments } \\ \text { EI } & : \text { Expected Improvement } \\ \text { PPM } & : \text { Parts Per Million } \\ \text { RANS } & : \text { Reynolds-averaged Navier Stokes } \\ \text { RSM } & \text { : Response Surface Model }\end{array}$

\section{ACKNOWLEDEMENT}

It is gratefully acknowledged that the work presented in this paper has been supported by Rolls-Royce plc, UK. We also thank Dr. David Toal and Dr. Alexander Forrester of University of Southampton for their inputs and discussion.

\section{REFERENCES}

[1] Advisory Council for Aeronautics Research in Europe, 2002, The Challenge of the Environment, Strategic Research Agenda, Vol. 2(3), European Commission, Belgium

[2] Lefebvre, A.H., 1999, Gas Turbine Combustion, Taylor and Francis, Philadelphia

[3] Wankhede, M. J., Bressloff, N. W., Keane, A. J., 2011, "Combustor Design Optimisation Using Co-Kriging of Steady and Unsteady Turbulent Combustion, Journal of Engineering for Gas Turbines and Power, GTP-11-1113, 133(12), (DOI: 10.1115/1.4004155)

[4] Jeong, S., Minemura, Y., Obayashi, S., 2006, "Optimisation of Combustion Chamber for Diesel Engine Using Kriging Model," Journal of Fluid Science and Technology, 1(2), 138-146

[5] Duchaine, F., Morel, T., Gicquel, L. Y. M., 2009, "Computational-Fluid-Dynamics-Based Kriging Optimization Tool for Aeronautical Combustion Chambers," AIAA Journal, Vol. 47/3 (DOI: 10.2514/1.37808)

[6] Correa, S.M., 1998, "Power Generation and Aero Propulsion Gas Turbines: From Combustion Science to Combustion Technology," Proc. Combust. Inst. 27, pp. 1793-1807

[7] Rolls-Royce, 2007, The Jet Engine, Key Publishing, UK

[8] Keller, J. O., Vaneveld, L., Korschelt, D., Hubbard, G. L., Ghoniem, A. F., Daily, J. W., Oppenheim, A. K., 1982, "Mechanism of Instabilities in Turbulent Combustion Leading to Flashback," AIAA Journal, Vol. 20, pp.254-262

[9] Claire, S., 2008, Gas Turbines: A Handbook of Air, Land and Sea Applications, Butterworth-Heinemann, Elsevier

[10] User's Guide, 2012, ANSYS FLUENT version 12.1

[11] Poinsot, T., Veynante, D., 2005, Theoretical and Numerical Combustion, Edwards Inc., USA

[12] Malte, P. C., Pratt, D. T., 1974, "Measurement of Atomic Oxygen and Nitrogen Oxides in Jet Stirred Combustion Engines," Proceedings of the Combustion Institute, 15:1061

[13] Hill, S. C., Smoot, L. D., "Modelling of Nitrogen Oxides Formation and Destruction in Combustion Systems," Progress in Energy and Combustion Science, 26:417-458

[14] http://www.3ds.com/products/catia

[15] Jones, D. R., 2001, “A Taxonomy of Global Optimization Methods Based on Response Surfaces," Journal of Global Optimization, 21/4, pp. 345-383

[16] Krige, D.G., 1951, “A Statistical Approach to Some Basic Mine Valuation Problems on the Witwatersrand," Journal of the Chemical, Metallurgical and Mining Engineering Society of South Africa, 52/6, pp.119-139

[17] Sacks, J., Welch, W. J., Mitchell, T. J., Wynn, H., P., 1989, "Design and Analysis of Computer Experiments," Statistical Science, Vol. 4/4, pp. 409-435

[18] Forrester, A. I. J., Keane, A. J., 2009, "Recent advances in surrogate-based optimization," Progress in Aerospace Sciences, 45(1-3): 50-79

[19] Forrester, A. I. J., Sóbester, A., Keane, A. J., 2008, Engineering Design via Surrogate Modelling: A Practical Guide, John Wiley and Sons, Chichester

[20] Kennedy, M. C., O' Hagan, A., 2000, "Predicting the Output From Complex Computer Code When Fast Approximations are Available," Biometrika, 87(1)

[21] Efron, B., Tibshirani R., 1993, An Introduction to the Bootstrap, Chapman and Hall, London

[22] Cumming G., Finch, S., 2005, "Inference by Eye: Confidence Intervals and How to Read Pictures of Data," American Psychologist, 60(2), pp. 170-180 